\title{
Microstructural analysis of conditioned and unconditioned responses to maltodextrin
}

\author{
DOMINIC M. DWYER \\ Cardiff University, Cardiff, Wales
}

\begin{abstract}
The microstructure of licking responses was analyzed to investigate the interaction between unconditioned responses to maltodextrin and the responses to flavor cues previously associated with maltodextrin. Experiment 1 demonstrated that although the consumption of maltodextrin peaked at intermediate concentrations, the mean lick cluster size showed a positive, monotonic increase with concentration. In Experiment 2, a (conditioned stimulus) CS + flavor was paired with $16 \%$ maltodextrin, whereas a CS - flavor was paired with $2 \%$ maltodextrin. During test, consumption of the CS + was higher than that of the CS - when the flavors were combined with $2 \%$ maltodextrin, but not when combined with $16 \%$ maltodextrin. In contrast, cluster size was larger with the $\mathrm{CS}+$ than with the $\mathrm{CS}-$, regardless of the concentration of maltodextrin present on test. Previous analyses of licking microstructure indicate that cluster size reflects the palatability of the ingested solution. Thus, the present results indicate that flavor conditioning can change the palatability of the cue flavors. Adding the CS + flavor to maltodextrin produced results analogous to increasing the concentration of maltodextrin (in terms of both consumption and licking microstructure measures), which is consistent with the idea that after conditioning, responses to the CS + flavor and to the unconditioned stimulus are mediated via the same representation.
\end{abstract}

Humans and other omnivorous animals demonstrate unlearned positive reactions to only a very few tastes. The reaction to the sweet taste of sugars is perhaps the most well documented; however, there is also abundant evidence not only that rodents can detect the taste of polysaccarides, but also that they find their taste attractive (see Sclafani, 1987, and Sclafani, 2004, for reviews). In addition, it is well established that pairing an otherwise neutral flavor with a substance that elicits an unconditioned reaction will endow that cue flavor with the ability to elicit a reaction of its own (see, e.g., Capaldi, 1992; Rozin \& Zellner, 1985; Sclafani, 1987). For example, rats will learn a preference for a flavor that is consumed along with glucose or fructose (e.g., Sclafani \& Ackroff, 1994), or for a flavor that is paired with the gastric infusion of nutrients (e.g., Sclafani \& Nissenbaum, 1988). Flavor preference learning of this type can be considered an example of Pavlovian conditioning: The flavor conditioned stimulus (CS) is paired with either the taste of the unconditioned stimulus or its postingestive consequences (US), and this cue-consequence association endows the CS flavor with the ability to elicit a behavioral response.

Pearce (2002) suggested that flavor preference learning might be based on a specifically configural learning process (see also Capaldi \& Hunter, 1994; Harris \& Thein, 2005). Pearce suggested that when two flavors are presented together, they should excite a configural representation of the flavor compound and that subsequent presentation of one flavor should (via activation of the configural unit) activate the sensory input unit of the other. To the extent that one flavor supports a positive reaction on the basis of palatability, the other should produce a similar conditioned reaction. Pearce also suggested that if one flavor contained nutrients, then a second associative link would also be important-namely, a direct association formed between the configural unit excited by the two flavors and the postingestive consequences of the nutrient. This network is illustrated in panel A of Figure 1. With respect both to responding on the basis of palatable tastes and to responding on the basis of the postingestive consequences of nutrients, Pearce's analysis suggests that conditioned and unconditioned responding should occur on the basis of the activation of the same representations: With respect to palatability, this is the sensory representation of the reinforcer and its automatic connection to a palatability-based response. With respect to nutrients, this is the configural unit representing the conjunction of the CS and US tastes and its acquired connection to the postingestive consequences of nutrients (although the sensory representation of the US should also be activated by the CS in this case). One implication of this idea is that conditioned and unconditioned reactions should interact, because the activation of the relevant reinforcer representation should be elicited both by the presentation of the reinforcer itself and by the

D. M. Dwyer, dwyerdm@cardiff.ac.uk 
A

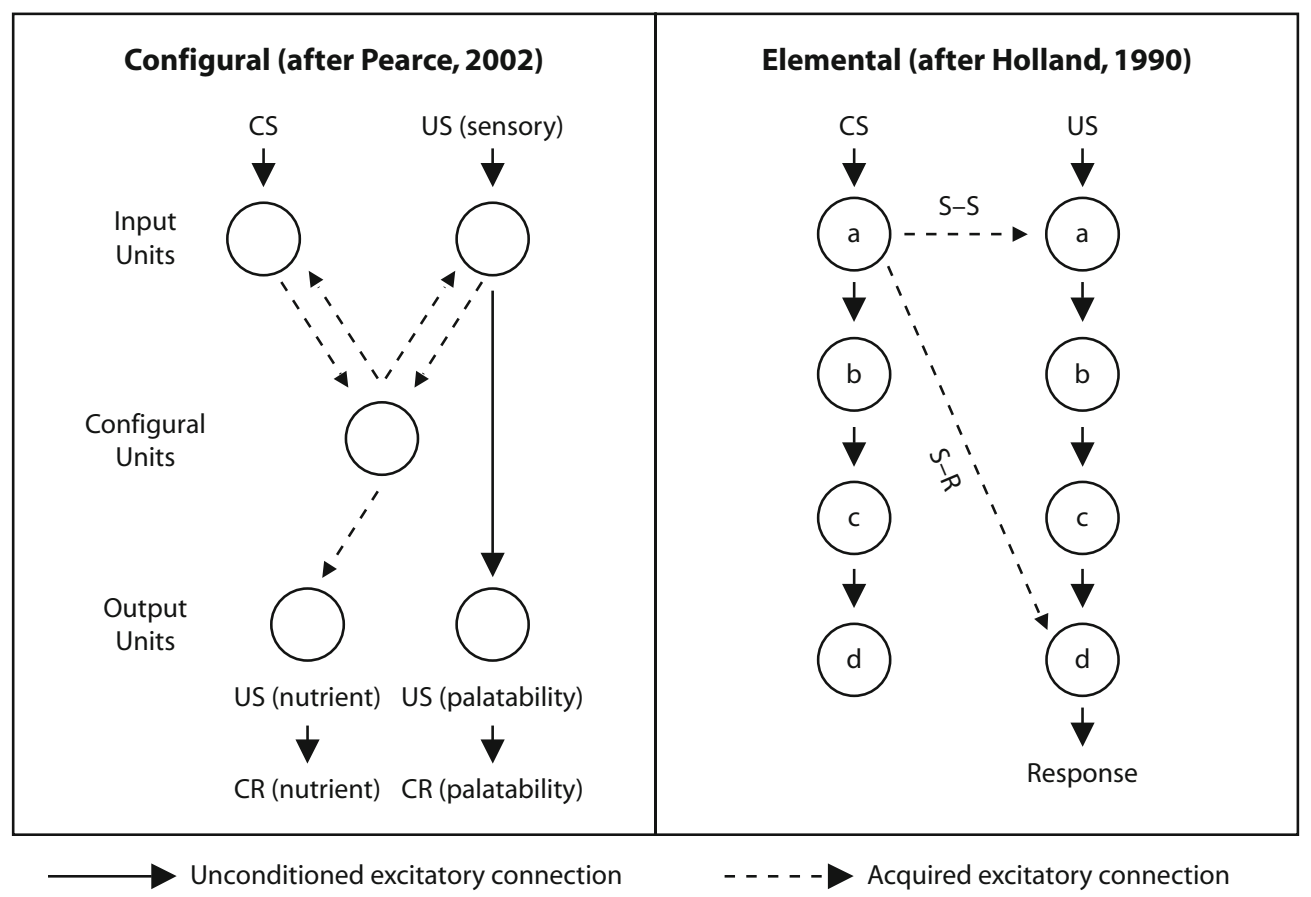

Figure 1. Examples of configural (A) and elemental (B) networks proposed to underpin flavor conditioning. In panel B, the nodes coded as " $a$ " to " $d$ " represent units activated by the CS and the US, beginning with purely sensory processing (a) and ending with motor processing controlling responding (d). NoteThe illustration in panel $A$ is from "Evaluation and Development of a Connectionist Theory of Configural Learning,” by J. M. Pearce, 2002, Animal Learning \& Behavior, 30, p. 87. Copyright 2002 by Psychonomic Society, Inc. Adapted with permission. The illustration in panel B is from "Event Representation in Pavlovian Conditioning: Image and Action," by P. C. Holland, 1990, Cognition, 37, p. 108. Copyright 1990 by Elsevier Ltd. Adapted with permission.

cue flavor that had been associated with it. Although the idea that conditioned and unconditioned reactions to the same substance should interact has been developed in the context of specifically configural mechanisms, elemental approaches can also be applied. For example, Holland (1990) provided a discussion of sensory-sensory associations that also envisages conditioned and unconditioned responses being mediated by the same sensory representations (see panel B of Figure 1 for an illustration of this network). Thus, both configural and elemental models can instantiate the idea that conditioned and unconditioned responses may be mediated via the same representation. This is not to argue that there are no differences between elemental and configural accounts of flavor conditioning, since Pearce (2002) is explicit in drawing a number of distinctions - especially with respect to the extinction of associations between the purely sensory aspects of the CS and US. In addition, Holland's ideas allow for the CS to activate overlapping representations of the US that do not involve co-activation of sensory representations (see the S-R connection in panel B of Figure 1), which raises the possibility that an interaction between conditioned and unconditioned responses need not always involve the activation of sensory aspects of the US by the CS.
In line with the idea that CSs should activate the same representations as the USs in flavor conditioning, it has been demonstrated that there is an interaction between rats' unconditioned responses to sucrose and their conditioned responses to flavors paired with sucrose (Harris \& Thein, 2005; Sclafani, 2002; Warwick, Synowski, Coons, \& Hendrickson, 1999; Warwick \& Weingarten, 1996). For example, having initially demonstrated that water-deprived rats preferred intermediate concentrations of sucrose, Harris and Thein subsequently demonstrated that rats preferred an odor previously associated with $30 \%$ sucrose to an odor previously associated with $5 \%$ sucrose when both odors were presented in 5\% sucrose. Their preference was reversed, however, when the odors were presented in $30 \%$ sucrose. Harris and Thein interpreted this pattern of results as reflecting the idea that the odor previously associated with $30 \%$ sucrose tasted sweet and that this sweetness summed with the taste of the sucrose solution in which the odor was presented: When the sucrose concentration was low, this pushed the summed sweetness into the most preferred intermediate range, but when the sucrose concentration was high, this pushed the summed sweetness into an unpreferred high range.

Although these results are certainly consistent with the idea that the conditioned and unconditioned responses to 
a taste are mediated via the same representation, they are all based on the analysis of consumption in one-bottle acceptance tests or two-bottle preference tests. Although simple consumption measures reflect some aspects of preference, it has long been recognized that total intake per se merely reflects the output of a variety of ingestive behaviors (see, e.g., Davis, 1998; Davis \& Levine, 1977; Davis \& Smith, 1992; Spector, Klumpp, \& Kaplan, 1998). This has led to the development of methods for the microstructural analysis of ingestive behavior and, in particular, for the analysis of the patterns of licking displayed by rats during the consumption of fluids. The ingestive behavior of rats consuming fluids consists of sustained runs of rapidly occurring rhythmic licks (referred to in the present article as clusters) separated by pauses of varying lengths. ${ }^{1}$ One consistent finding in the literature examining the consumption of solutions that elicit positive unconditioned reactions is that the mean number of licks in a cluster bears a positive, monotonic relationship to the concentration of the ingested fluid. This relationship has led to the suggestion that cluster size can be used as an index of stimulus palatability (see, e.g., Davis, 1996; Davis \& Perez, 1993; Davis \& Smith, 1992; Spector et al., 1998). Importantly, cluster size is not directly related to the amount of solution consumed, since animals typically exhibit an inverted U-shaped function between concentration and total consumption, with the highest levels of consumption at intermediate levels of concentration. Cluster size also decreases monotonically with increasing concentration of solutions assumed to be unpalatable, such as quinine (e.g., Hsiao \& Fan, 1993; Spector \& St. John, 1998). The idea that cluster size should be considered to directly reflect palatability receives further support from the finding that pharmacological treatments thought to influence palatability also have a direct effect on cluster size (e.g., Asin, Davis, \& Bednarz, 1992; Higgs \& Cooper, 1998).

To my knowledge, the only previous application of microstructural analyses to conditioned flavor preference was by Myers and Sclafani (2001a), who reported that during the consumption of a CS flavor paired with the infusion of glucose to the stomach, cluster size was higher than during consumption of a CS flavor paired with infusion of water. This result implies that flavor preference learning not only can change the relative preference for otherwise neutral cue solutions, but also can produce a conditioned change in the palatability of those cue solutions. In a companion article, Myers and Sclafani (2001b) reported that flavor preference learning also produces a change in cue palatability, as indexed by rats' orofacial reactions (the so-called taste reactivity test-Grill \& Norgren, 1978). Thus, the analysis of licking microstructure - and, in particular, the variable of mean cluster size - has been shown to be sensitive to both conditioned and unconditioned differences in the palatability of test solutions. However, there has been no investigation of how conditioned and unconditioned palatability responses interact. There is good evidence from the analysis of consumption alone that Pearce (2002) is correct in his suggestion that conditioned and unconditioned flavor preferences are mediated by the same representation. The present study sought to build on previous investigations of the interaction between conditioned and unconditioned reactions to palatable flavors by using microstructural analysis methods to examine whether the interaction between the consumption of a palatable taste - and the neutral flavor previously paired with it - extends to palatability reactions, as indexed by cluster size.

\section{EXPERIMENT 1}

There is abundant evidence that rats find the taste of polysaccharides palatable, although humans do not tend to find them so (see Sclafani, 1987, and Sclafani, 2004, for reviews). However, there is only one previous report of a microstructural analysis of licking behavior during the consumption of polysaccharide solutions. Davis (1996) analyzed the behavior of rats consuming different concentrations of polycose (a commercially available product containing a mixture of polysaccharides, along with small quantities of mono- and disaccharides). He observed that cluster size was positively related to the concentration of polycose, indicating that - as with simple sugars - the palatability of polysaccarides increases with concentration. However, rats were tested in the absence of food deprivation, and previous studies examining the palatability of sucrose have revealed inconsistent effects of food deprivation on palatability responses. For example, Davis and Perez (1993) reported an interaction between food deprivation and sucrose concentration in which food deprivation increased the palatability (as indexed by cluster size) of high but not of low sucrose concentrations, whereas Spector et al. (1998) reported no effects of deprivation on cluster size. Experiment 2 was aimed at analyzing the interaction between conditioned and unconditioned responses to maltodextrin in food-deprived rats. Thus, Experiment 1 examined the unconditioned responses of food-deprived rats to different concentrations of maltodextrin, because there is no previous report of licking microstructure analysis of animals consuming polysaccharides when they are food deprived.

\section{Method}

\section{Subjects and Apparatus}

Eight male hooded Lister rats (supplied by OLAC, Bicester, U.K.) were used in Experiment 1. All were housed in pairs under a 12:12-h light:dark cycle. They had free-feeding weights in the range of 305-324 g. During testing, the rats' food ration was adjusted to maintain them between $85 \%$ and $90 \%$ of their free-feeding weights. This food ration was given in the home cages $1 \mathrm{hr}$ after the daily experimental session had been completed. Throughout the experiment, drinking sessions began at approximately 11 a.m.

Training and testing took place in a separate experimental room that contained six automated drinking chambers (Med Associates, Inc., St. Albans, VT) measuring $30 \times 24 \times 21 \mathrm{~cm}$. The floor was 19 steel rods: $4.8 \mathrm{~mm}$ in diameter, $16 \mathrm{~mm}$ apart. Two walls were clear Perspex, and two were aluminum. There were two $2 \times 1 \mathrm{~cm}$ holes approximately $5 \mathrm{~cm}$ above the grid floor in one of the aluminum 
walls - one on each side of the cage. Fluids could be made available through stainless-steel drinking spouts attached to 50-ml cylinders. These cylinders were mounted on devices that automatically positioned the drinking spout level with the front of the cage and retracted it at the end of the session. Only the left-hand spout was used during Experiment 1. A contact-sensitive lickometer registered the time of each lick to the nearest $0.01 \mathrm{sec}$, and a microcomputer running MED-PC software (Med Associates, Inc.) controlled the equipment and recorded the data.

Consumption was assessed by weighing the bottles before and after each session. The solutions used in Experiment 1 were $2 \%, 4 \%, 8 \%$, and $16 \%(\mathrm{w} / \mathrm{w})$ maltodextrin (C*Dry MD 01904 , Cerestar-UK, Manchester, U.K.), made up in deionized water. Note that the maltodextrin used in the present experiment contained approximately $2 \%$ mono- and disaccharides, with the remainder being polysaccharides.

\section{Procedure}

Previous training. Before being used in Experiment 1, the animals were used in a study investigating blocking of flavor preference conditioning in the same apparatus as was used in the present article. This meant that prior to the start of the present experiment, the animals had received exposure to both high (16\%) and low (2\%) concentrations of fructose, both in compound with various Kool-Aid flavors (Kraft Foods USA, Rye Brook, NY) while they were food deprived. At the completion of this experiment, the animals were allowed free food access for 3 days before being returned to food deprivation.

Palatability testing. All testing sessions were $30 \mathrm{~min}$ in duration, and there was one test session per day. Half of the animals received exposure to $2 \%, 4 \%, 8 \%$, and $16 \%$ maltodextrin in ascending order of concentration across days, and the other half received exposure in descending order.

Data analysis. In addition to the daily consumption measurement, three parameters were extracted from the record of licks: number of clusters, number of licks, and the mean cluster size. A cluster was defined as a set of licks, each separated by an interlick interval of no more than $0.5 \mathrm{sec}$. This is the criterion adopted by Davis and his coworkers (see, e.g., Davis \& Perez, 1993; Davis \& Smith, 1992), and, although other criteria have been used (e.g., $1 \mathrm{sec}$ by Spector et al., 1998), parametric analyses suggest that there is little practical difference between them since most pauses greater than $0.5 \mathrm{sec}$ are also greater than $1 \mathrm{sec}$ (e.g., Davis \& Smith, 1992; Spector et al., 1998). Repeated-measures ANOVAs were used to analyze the data, with all statistical tests reported in the present article using a significance value of $\alpha=.05$.

\section{Results and Discussion}

Figure 2 shows the mean amount of fluid consumed (panel A) and mean cluster size (panel B), as well as the total number of licks (panel C) and clusters (panel D) as a factor of maltodextrin concentration. The statistical analysis of these variables will be described in turn. An ANOVA revealed a significant effect of concentration on consumption $[F(3,21)=32.5, p<.001]$. Pairwise comparisons were used to explore this effect and revealed that although consumption of $8 \%$ maltodextrin was nu-
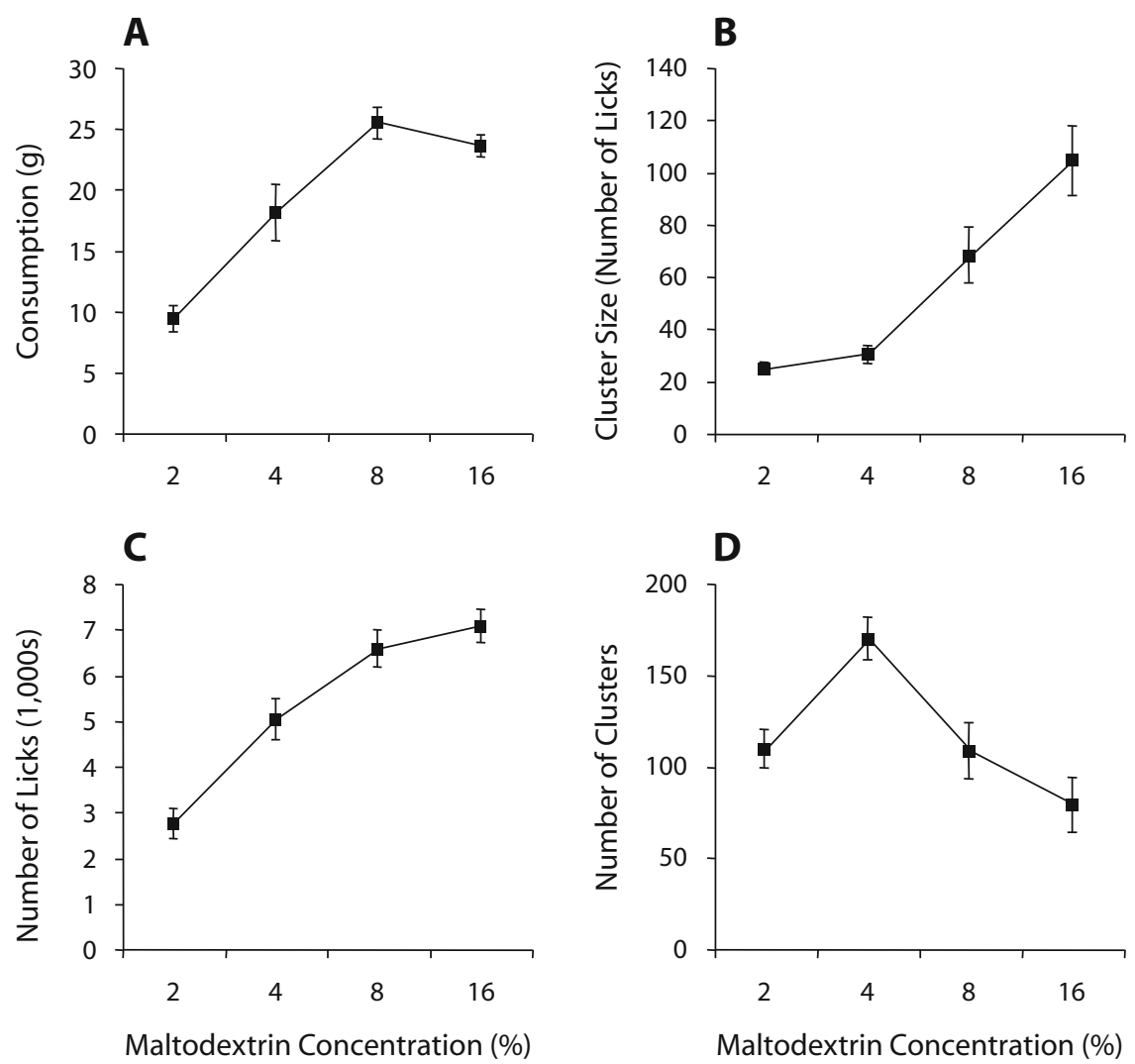

Figure 2. The data from Experiment 1 as a function of maltodextrin concentration: (A) mean consumption; (B) mean number of licks per cluster; (C) total licks per session; (D) total number of clusters. Bars represent SEMs. 
merically higher than that of $16 \%$, the difference was not significant $[t(7)=1.8, p=.115]$. The consumption of $8 \%$ maltodextrin was higher than that of $2 \%$ and $4 \%$ [lowest $t(7)=3.3, p=.013$ ]. The difference between the consumption of $4 \%$ and $16 \%$ maltodextrin only approached conventional significance levels $[t(7)=2.4$, $p=.050]$, but consumption of $16 \%$ maltodextrin was higher than that of $2 \%[t(7)=9.3, p<.001]$. The consumption of $2 \%$ maltodextrin was also lower than that of $4 \%[t(7)=5.1, p<.001]$. This pattern of results indicates that consumption was highest at intermediate to high concentrations of maltodextrin. An ANOVA revealed a significant effect of concentration on cluster size $[F(3,21)=25.6, p<.001]$. An exploration of this effect with pairwise comparisons revealed that cluster size was higher with $16 \%$ maltodextrin than with all other concentrations [lowest $t(7)=3.2, p=.016$ ], that cluster size was higher with $8 \%$ maltodextrin than with $2 \%$ or $4 \%$ [lowest $t(7)=4.0, p=.005$ ], but that cluster size did not differ significantly between $2 \%$ and $4 \%$ maltodextrin $[t(7)=1.4, p=.197]$. In contrast to the consumption data, this pattern of results indicates that cluster size increased monotonically with concentration. An ANOVA also revealed a significant effect of concentration on the total number of licks $[F(3,21)=47.3, p<$ $.001]$. Pairwise comparisons revealed that there was no difference in the number of licks for $8 \%$ and $16 \%$ maltodextrin $[t(7)=1.3, p=.242]$, and the number of licks for either $8 \%$ or $16 \%$ maltodextrin was higher than that of all other concentrations [lowest $t(7)=4.1, p=.005$ ]. There were also fewer licks for $2 \%$ than for $4 \%$ maltodextrin $[t(7)=7.2, p<.001]$. Thus, the lick data largely mirror the consumption data supporting the idea that consumption was highest at intermediate to high concentrations. Finally, an ANOVA revealed a significant effect of concentration on the number of clusters $[F(3,21)=$ $8.95, p=.001]$, and pairwise comparisons revealed that the number of clusters was higher with $4 \%$ maltodextrin than with all other concentrations [lowest $t(7)=2.9$, $p=.023]$, that the number of clusters was higher with $2 \%$ maltodextrin than with $16 \%[t(7)=2.9, p=.022]$, but that the number of clusters for $8 \%$ maltodextrin did not differ from that of $2 \%$ or $16 \%$ maltodextrin [highest $t(7)=1.6, p=.155]$.

In summary, the present results indicate that the concentration of maltodextrin was monotonically related to cluster size in food-deprived animals. This replicates results observed with nondeprived animals (Davis, 1996) and is exactly what would be expected, given that cluster size has been identified as a parameter directly related to stimulus palatability (see, e.g., Davis \& Smith, 1992; Spector et al., 1998). Previous studies have also reported an inverted U-shaped relationship between the concentration of maltodextrin and consumption in real feeding conditions (e.g., Davis, 1996; Nissenbaum \& Sclafani, 1987). It is probable that postingestive satiety limits the consumption of high maltodextrin concentrations because under sham-feeding conditions, rats actually do consume more $16 \%$ maltodextrin than they do $8 \%$ (Nissenbaum \& Sclafani, 1987). The present data are consistent with these observations, although there was no direct evidence for a reduction in consumption at concentrations above $16 \%$ maltodextrin, since the range of concentrations tested was lower than that in previous studies.

\section{EXPERIMENT 2}

The results of Experiment 1 confirmed that the palatability of maltodextrin solutions is positively related to their concentration but that maximum levels of consumption are seen at intermediate concentrations. The present experiment had two aims. The first was to confirmusing maltodextrin as the reinforcer- that flavor preference learning can indeed produce a conditioned change in the palatability of previously neutral cue flavors. The second aim was to investigate the effects of combining different concentrations of maltodextrin with cue flavors previously paired with maltodextrin, in terms of both consumption and palatability responses. Therefore, Experiment 2 began by training a flavor preference via pairing one flavor with $16 \%$ maltodextrin (the $\mathrm{CS}+$ ) and a second flavor with $2 \%$ maltodextrin (the $\mathrm{CS}-$ ). These training parameters have previously been shown to result in preferential consumption of the CS+ over the CSflavor when both flavors were combined with $2 \%$ maltodextrin in two-bottle tests (Dwyer \& Quirk, in press). The consumption of each of the CS + and CS - was then examined with one-bottle tests under two conditions: once when mixed with $2 \%$ maltodextrin and once when mixed with $16 \%$ maltodextrin. If it is assumed that conditioned and unconditioned responses to maltodextrin are mediated through the same representation, then the response to the CS + should be similar to that produced by increasing the concentration of maltodextrin. Given that maximum consumption is seen with intermediate concentrations of maltodextrin, greater consumption of the $\mathrm{CS}+$ than of the CS - should be seen against the background of $2 \%$ maltodextrin, but not of $16 \%$. Given that the palatability responses are positively related to increasing concentrations of maltodextrin, the palatability of the CS + should be higher than that of the CS - against the background of both $2 \%$ and $16 \%$ maltodextrin.

\section{Subjects and Apparatus}

\section{Method}

Sixteen naive, male hooded Lister rats were used in Experiment 2, with free-feeding weights in the range of 336-386 g at the start of the experiment. Animals were from the same source and were held under the same conditions as those described for Experiment 1. Experiment 2 was run using the same equipment used in Experiment 1. Grape and cherry Kool-Aid flavors at $0.05 \%$ (w/w) were used as the CSs. During training, the CS + flavor was combined with $16 \%$ maltodextrin, and the CS- was combined with $2 \%$ maltodextrin. During testing, animals received separate sessions in which both the CS + and CS - were combined with $2 \%$ and $16 \%$ maltodextrin.

\section{Procedure}

Preference training. All rats received two sessions of preliminary training. During each, they were placed in the drinking cages for 30 min with unlimited access to $2 \%$ maltodextrin. Throughout 
Table 1

Mean and Standard Error of the Mean (SEM) for Consumption (g), Total

Number of Licks, Total Number of Clusters, and Cluster Size (Number

of Licks) for the Final CS+ and CS- Training Days in Experiment 2

\begin{tabular}{|c|c|c|c|c|c|c|c|c|}
\hline \multirow[b]{2}{*}{ Solution } & \multicolumn{2}{|c|}{ Consumption } & \multicolumn{2}{|c|}{ Total Licks } & \multicolumn{2}{|c|}{ Total Clusters } & \multicolumn{2}{|c|}{ Cluster Size } \\
\hline & $M$ & $\overline{S E M}$ & $M$ & SEM & $M$ & SEM & $M$ & SEM \\
\hline $\mathrm{CS}$ & 26.0 & 007 & 7 & & & & 56.56 & 2.96 \\
\hline $\mathrm{CS}-$ & 12.39 & 0.97 & $2,720.21$ & 174.96 & 166.64 & 12.04 & 17.39 & 1.67 \\
\hline
\end{tabular}

the experiment, drinking sessions began at approximately 11 a.m All rats received 8 days of training, during which the CS + flavor was paired with $16 \%$ maltodextrin and the CS - flavor was paired with $2 \%$ maltodextrin. The identity of the CS+ and CS - flavors was counterbalanced among rats. Training days alternated between the $\mathrm{CS}+$ and $\mathrm{CS}-$, with half of the rats receiving the CS + flavor on the first training day and the other half receiving the $\mathrm{CS}-$. All sessions were $30 \mathrm{~min}$ long, and rats were allowed unlimited access to the cue flavors. These trials were repeated in order until each cue flavor had been presented four times.

Test. After preference training was completed, a 4-day testing procedure began with one 30-min test conducted on each day. All animals received two test sessions with the $\mathrm{CS}+$ : once combined with $16 \%$ maltodextrin and once combined with $2 \%$. They also received two test sessions with the CS-, once combined with $16 \%$ maltodextrin and once combined with $2 \%$. Half of the animals received the $16 \%$ tests before the $2 \%$ tests, and the order was reversed for the remaining animals. Within each group of rats, half received the $\mathrm{CS}+$ tests before the CS - tests, and the order was reversed for the remaining animals.

Data analysis. As in Experiment 1, four parameters were subjected to analysis: consumption, number of clusters, number of licks, and the mean cluster size. Clusters were defined in the same way as they were in Experiment 1. The data for two animals were removed from the analysis: one because of a leaking spout during the $2 \% \mathrm{CS}+$ test and the other because of a blocked spout during the $2 \% \mathrm{CS}-$ test. However, the inclusion of one or both of these animals in the final analysis would have had little material impact on the results.

\section{Results and Discussion}

The data from the final training session with the CS+ and $\mathrm{CS}-$ are shown in Table 1 . The rats consumed more of the $\mathrm{CS}+$ than of the $\mathrm{CS}-[t(13)=9.3, p<.001]$, produced more licks to the $\mathrm{CS}+[t(13)=11.5, p<.001]$ than to the $\mathrm{CS}-$, but produced fewer clusters to the $\mathrm{CS}+$ $[t(13)=-4.7, p<.001]$. Consequently, the cluster size was greater for the CS + flavor $[t(13)=11.8, p<.001]$, reflecting the greater palatability of the CS + and $16 \%$ maltodextrin combination over the CS- and 2\% maltodextrin combination. The critical data from the test phase of Experiment 2 are shown in Figure 3. This figure shows the mean amount of fluid consumed (panel A) and mean cluster size (panel B), as well as the total number of licks
A

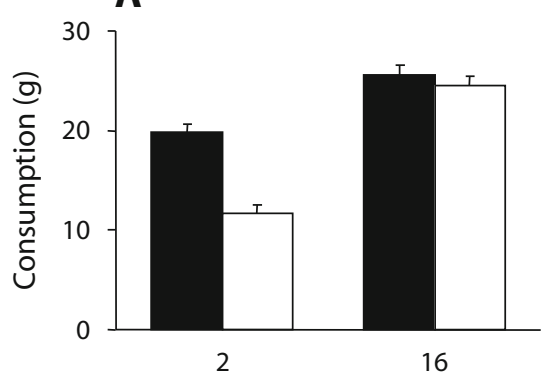

C

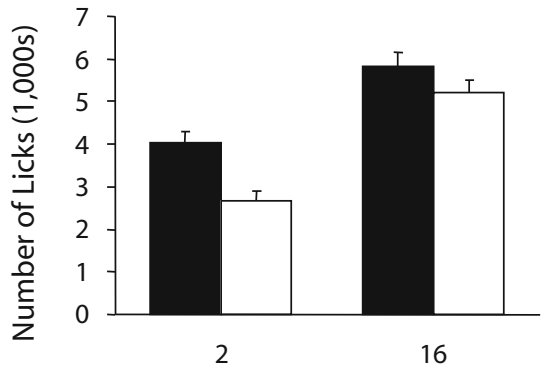

Maltodextrin Concentration (\%)
B

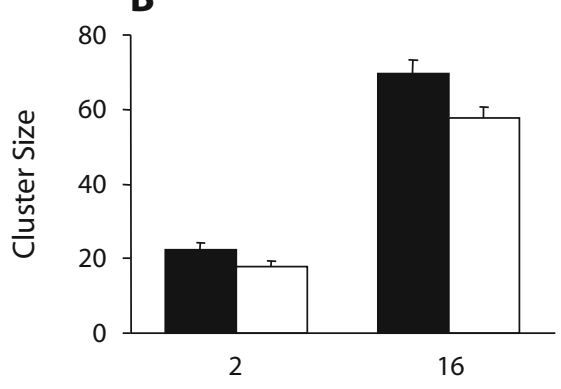

D

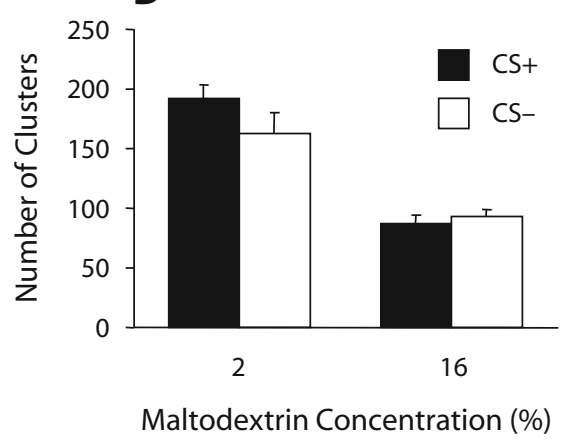

Figure 3. The test session data from Experiment 2 as a function of both cue type (black bars $=\mathrm{CS}+$; white bars $=\mathrm{CS}-$ ) and maltodextrin concentration: (A) mean consumption; (B) mean number of licks per cluster; (C) total licks per session; (D) total number of clusters. Bars represent SEMs. 
(panel C) and clusters (panel D) for both $2 \%$ and $16 \%$ maltodextrin concentrations, and for both $\mathrm{CS}+$ and CSflavors. An inspection of panel A indicates that consumption was higher with $16 \%$ than with $2 \%$ maltodextrin and that consumption of the CS+ was higher than that of the CS - only in the $2 \%$ maltodextrin tests. An inspection of panel B indicates that cluster size was higher with $16 \%$ than with $2 \%$ maltodextrin and that cluster size with the CS+ was higher than that with the CS - in both the $2 \%$ and $16 \%$ maltodextrin tests (albeit the difference was larger in the $16 \%$ tests). Panel $\mathrm{C}$ indicates that the total number of licks was higher with $16 \%$ than with $2 \%$ maltodextrin and that the number of licks with the $\mathrm{CS}+$ was higher than that with the CS - especially in the $2 \%$ maltodextrin tests. Panel D indicates that the total number of clusters was higher with $2 \%$ maltodextrin than with $16 \%$. There was a trend toward greater numbers of clusters with the CS+ in the $2 \%$ maltodextrin tests, but no such trend in the $16 \%$ tests.

The data were analyzed using a within-subjects ANOVA with effects of CS and maltodextrin concentration. With respect to consumption, the ANOVA revealed significant effects of concentration $[F(1,13)=93.0, p<.001]$ and CS $[F(1,13)=27.1, p<.001]$, as well as a significant interaction between them $[F(1,13)=22.1, p<.001]$. An analysis of simple main effects revealed that the difference between the CS+ and CS - was significant for the $2 \%$ maltodextrin tests but not for the $16 \%$ maltodextrin tests $[F(1,13)=49.0, p<.001$, and $F<1$, respectively $]$. With respect to cluster size, there were significant effects of concentration $[F(1,13)=148.4, p<.001]$ and $\operatorname{CS}[F(1,13)=$ $11.5, p=.005]$, but no significant interaction between them $[F(1,13)=1.8, p=.206]$. An analysis of simple main effects revealed that the difference between the CS + and CS - was significant for both the $2 \%$ and $16 \%$ maltodextrin tests $[F(1,13)=5.8, p=.031$, and $F(1,13)=6.1, p=.028$, respectively]. With total number of licks, there were significant effects of concentration $[F(1,13)=98.8, p<.001]$ and $\operatorname{CS}[F(1,13)=15.9, p=.002]$, as well as a significant interaction between them $[F(1,13)=9.51, p=.009]$. An analysis of simple main effects revealed that the difference between the CS + and CS - was significant for the $2 \%$ maltodextrin tests; however, in the $16 \%$ maltodextrin tests, the difference only approached the standard significance level $[F(1,13)=30.1, p<.001$, and $F(1,13)=4.2$, $p=.062$, respectively]. Finally, with respect to the number of clusters, there was a significant effect of concentration $[F(1,13)=54.1, p<.001]$, but no effect of $\operatorname{CS}[F(1,13)=$ $2.1, p=.169]$, nor was there a significant interaction between concentration and CS $[F(1,13)=3.4, p=.089]$.

The fact that the cluster size was larger with the $\mathrm{CS}+$ than with the CS - during test implies that the palatability of the $\mathrm{CS}+$ had been increased as a result of the previous pairing of the CS+ flavor with $16 \%$ maltodextrin. The size of the lick cluster difference between CS + and CS - was smaller than that reported previously when intragastric infusions of glucose were used as the US (Myers \& Sclafani, 2001a). However, in that study, animals continued to receive the glucose infusions during consumption of the $\mathrm{CS}+$. Thus, the present study confirms that the change in cluster size produced by flavor preference conditioning is not restricted to the use of intragastric glucose as the US, and it can be seen when the CSs are tested in extinction. Although the interaction between maltodextrin concentration and CS type was not significant, it is noticeable that the numerical magnitude of the cluster size difference was larger in the $16 \%$ than in the $2 \%$ maltodextrin tests. This result is consistent with those of Experiment 1, in which the slope of the curve relating cluster size to concentration was shallower at lower concentrations than at higher ones (see Davis, 1996, for similar results when rats are not food deprived).

Consumption of the CS + was higher than that of the CS - only when the concentration of maltodextrin was low. This is in direct contrast to the cluster size results that indicate that the palatability of the CS + was higher than that of the CS - for both high and low test concentrations of maltodextrin. In Experiment 1, increasing the concentration of maltodextrin beyond a certain point failed to produce further increases in consumption; however, increasing the concentration of maltodextrin produced monotonic increases in palatability. The present results are consistent with the pattern of results observed for Experiment 1 , if one makes the assumption that conditioned and unconditioned reactions to maltodextrin are mediated by the same representation. This idea will be examined in the General Discussion. Incidentally, the dissociation between the pattern of results seen with consumption and that seen with cluster size reinforces the idea that cluster size is an independent assay of palatability and not merely a reflection of the tendency to consume greater amounts of a palatable solution under certain circumstances.

\section{GENERAL DISCUSSION}

If the conditioned response to a flavor paired with a particular taste is mediated via the same representation as that of the unconditioned response to that taste, then conditioned and unconditioned responses should interact. The idea that conditioned and unconditioned responses should interact rather than be independent of each other was developed by Pearce (2002), who suggested that after the simultaneous pairing of two flavors, the presentation of one flavor will activate the sensory representation of the other. The idea that conditioned and unconditioned reactions should interact was examined through the analysis of both consumption and the pattern of licking responses. Considering consumption first, when both the CS + (previously paired with $16 \%$ maltodextrin) and CS - (previously paired with $2 \%$ maltodextrin) were presented with $2 \%$ maltodextrin during test, rats consumed more of the $\mathrm{CS}+$ flavor: This result reflects the fact that at low concentrations, increasing the concentration of maltodextrin resulted in higher consumption. However, when the CS+ and CS - were presented with $16 \%$ maltodextrin, there was no difference in the consumption of the CS+ and $\mathrm{CS}-$ : This result reflects the fact that at higher concentrations, increasing the concentration of maltodextrin did not result in greater consumption. Importantly, these consumption data - and similar, previous results (see, e.g., 
Harris \& Thein, 2005)—do not actually fully confirm Pearce's idea that it is a specifically sensory representation that is excited by both the CS and the US. As was noted in the introduction, the volume ingested across a whole session is not a direct measure of any individual response. It is actually a reflection of an aggregation of all of the different behaviors that occur during the time that the animal is drinking (see, e.g., Davis, 1998; Davis \& Levine, 1977; Davis \& Smith, 1992; Spector et al., 1998). These different responses could reflect the activity of a number of different representations, both sensory and otherwise. Thus, although the interaction between the amount consumed of conditioned and unconditioned cues for maltodextrin is certainly consistent with the idea that the same representation is activated by both the CS and $\mathrm{US}$, it is not uniquely consistent with the idea that this is a specifically sensory representation.

As was reviewed in the introduction, a variety of investigators have provided evidence in support of the idea that the size of licking clusters reflects the palatability of the test solution. It has also been argued that in the absence of any differences in motivational state or individual differences, this palatability measure reflects sensory experience. This idea, however, is far from being universally accepted (see, e.g., Davis, 1998; Davis \& Levine, 1977). In Experiment 2, the palatability (as indexed by the size of licking clusters) of the $\mathrm{CS}+$ was higher than that of the $\mathrm{CS}-$, regardless of whether they were mixed with $2 \%$ or $16 \%$ maltodextrin. If it is assumed that the size of licking clusters is related to the activation of purely sensory representations-and that the interaction of such palatability responses to conditioned and unconditioned stimuli suggest the activation of a single representation - then the lick-cluster results from Experiment 2 are in accordance with Pearce's (2002) suggestion that the expression of flavor preference learning is mediated via the activation of the same representation that is excited by the presentation of the reinforcer itself. Furthermore, this representation is a specifically sensory one. However, there are several aspects of the present study that question the uncritical acceptance of Pearce's analysis.

Pearce (2002) implied that pairing a CS with a taste will result in the CS's being able to elicit some of the sensory qualities of the taste - an idea that reflects the fact that after being paired with sucrose, odors are perceived to be sweet (see, e.g., Stevenson, Boakes, \& Wilson, 2000). Flavor preference conditioning can also modify the palatability of the cue flavors when intragastric infusions of glucose are used as the US (Myers \& Sclafani, 2001a, 2001b). It should be noted that this method of delivering the US prevents the animals from tasting it and thus presumably also prevents any rich sensory representation of glucose being activated or associated with the CS. Thus, the CS does not have to activate sensory qualities of the US to change the palatability of the CS. Therefore, the fact that the conditioned palatability reaction to the CS+ in Experiment 2 interacted with the unconditioned palatability reaction to the US does not, in isolation, prove that the interaction is the result of both the CS and US activating a common sensory representation.

Although maltodextrin has a palatable taste to rats (Sclafani, 1987), there is little evidence that the taste of maltodextrin alone is sufficient to support flavor preference learning in the absence of postingestive nutrient effects. Indeed, it has previously been reported that when the carbohydrate digestion is blocked, maltodextrin does not support flavor preference learning (Elizalde \& Sclafani, 1988). Unpublished data from Sclafani's laboratory suggest that under sham-feeding conditions, sucrose - but not polycose-will condition a preference for the $\mathrm{CS}+$. The data further suggest that in animals fed ad lib, $2 \%$ sucrose - but not $2 \%$ polycose-will condition a preference for the $\mathrm{CS}+$ (personal communication, January 14, 2008). Taken together, these results suggest that in the absence of postingestive nutrient effects, maltodextrin is ineffective as a reinforcer in flavor conditioning. The mechanism by which maltodextrin acts as a reinforcer is highlighted by a comparison to fructose, which does not condition a preference in brief sessions when infused directly into the stomach or when there is a delay between the CS and US (Sclafani \& Ackroff, 1994; Sclafani, Cardieri, Tucker, Blusk, \& Ackroff, 1993; Sclafani, Fanizza, \& Azzara, 1999). Thus, any flavor preference conditioned by fructose is attributable to its palatable taste alone. The fact that Dwyer and Quirk (in press) found a dissociation between performance on a context-conditional flavor preference (when fructose and maltodextrin were used as the reinforcers) implies that they do not both depend on the same reinforcing action. Thus, it is highly unlikely that the conditioned responses to the $\mathrm{CS}+$ in the present study were actually due to the activation of a representation of the sensory aspects of maltodextrin. Instead, in the terms of the network described in panel A of Figure 1, the most relevant pathway for the conditioned response is that via the configural unit to the representation of the postingestive consequences of the nutrient. The same pathway should be activated by the presentation of maltodextrin itself, thus supporting the interaction of conditioned and unconditioned responses. In terms of the elemental network outlined in panel B of Figure 1, the relevant pathway would be that via the learned S-R association leading to the activation of the US pathway remote from sensory experience. Thus, whether conceived in configural or elemental terms, the lick-cluster size analysis of palatability is consistent with the idea that the same representation is activated by both the CS and the US. But instead of this co-activated representation being specifically sensory (as was originally suggested by Pearce, 2002), the present results are more consistent with the co-activation of some representation of the postingestive consequences of nutrients.

Thus far, the observed interactions between the conditioned and unconditioned responses (in terms of both consumption and palatability) have been examined only in light of the idea that the level of responding reflects the degree to which the representation of maltodextrin is 
activated. However, the CS + was paired with $16 \%$ maltodextrin and the $\mathrm{CS}-$ was paired with $2 \%$ maltodextrin during training in all animals. Thus, rather than $2 \%$ and $16 \%$ maltodextrin producing less or more activation of a single representation, the animals may actually come to represent $2 \%$ and $16 \%$ maltodextrin entirely separately. During testing against the $16 \%$ maltodextrin background, the CS - should activate a representation of $2 \%$ maltodextrin. Since $16 \%$ maltodextrin may also be presumed to activate a representation of itself, this condition will result in two representations of maltodextrin being concurrently activated: a weak one and a strong one. In comparison, during the $\mathrm{CS}+$ test, only the representation of the strong maltodextrin should be activated. It is possible that the activation of the representations of both weak and strong maltodextrin might produce a conflict that disrupts responding. Although it is not immediately obvious why this conflict should have a greater impact on palatability responses than on consumption responses (because the consumption and palatability of $2 \%$ maltodextrin were both below that of $16 \%$ maltodextrin in Experiment 1), it is, of course, possible that the lack of a consumption difference between $\mathrm{CS}+$ and $\mathrm{CS}$ - was due to a ceiling effect. In the test against a $2 \%$ background, the CS + should result in the activation of both strong and weak representations of maltodextrin. In the CS - case, only the weak one is activated; thus, although concurrent activation of strong and weak maltodextrin representations should also produce conflict, the fact that there was at least some activation of the strong maltodextrin representation should result in greater responding to the CS+ in terms of both consumption and palatability. Although this account does not require that weak and strong maltodextrin share the same representation, it still assumes that the presentation of a CS flavor will activate a representation of the reinforcer with which it was previously paired. Therefore, this account also relies on the idea that both the CS+ and $16 \%$ maltodextrin should activate the same representation - albeit not by the same mechanism as was discussed previously.

In conclusion, adding the CS + flavor to maltodextrin produced results that were analogous to increasing the concentration of maltodextrin. This interaction between the responses to flavor cues previously paired with maltodextrin and the unconditioned responses to maltodextrin itself is consistent with the idea that flavor conditioning allows the CS to activate the same representations as the US itself. The fact that there was an interaction between conditioned and unconditioned reactions in terms of both consumption and palatability measures indicates that a range of behaviors are controlled by this shared representation. Although the present data are consistent with Pearce's (2002) suggestion that a configural representation of the CS and US is formed (allowing the CS to activate the sensory aspects of the US), they are also consistent with elemental accounts. Furthermore, there is reason to believe that the reinforcing action of maltodextrin depends more on its nutritional components than on its sensory components. Thus, a conclusive answer to the question of whether flavor conditioning is best described either by specifically configural or elemental processes will require further experimental work.

\section{AUTHOR NOTE}

The experimental work reported in the present article was supported by a BBSRC New Investigator Award to D.M.D. The author also thanks an anonymous reviewer of an earlier version of this article for the suggestion that weak and strong maltodextrin could be represented separately; thus, differential responding might be under the control of the CS - . Address correspondence to D. M. Dwyer, School of Psychology, Cardiff University, Tower Building, Park Place, Cardiff CF10 3AT, Wales (e-mail: dwyerdm@cardiff.ac.uk).

\section{REFERENCES}

Asin, K. E., Davis, J. D., \& Bednarz, L. (1992). Differential effects of serotonergic and catecholaminergic drugs on ingestive behavior. Psychopharmacology, 109, 415-421.

Capaldi, E. D. (1992). Conditioned food preferences. In D. L. Medin (Ed.), The psychology of learning and motivation (Vol. 28, pp. 1-33). San Diego: Academic Press.

CAPAldi, E. D., \& Hunter, M. J. (1994). Taste and odor in conditioned flavor preference learning. Animal Learning \& Behavior, 22, 355-365.

DAvis, J. D. (1996). Microstructural analysis of the ingestive behavior of the rat ingesting polycose. Physiology \& Behavior, 60, 1557-1563.

DAVIS, J. D. (1998). A model for the control of ingestion-20 years later. In S. J. Fluharty \& A. R. Morrison (Eds.), Progress in psychobiology and physiological psychology (Vol. 17, pp. 127-173). San Diego: Academic Press.

Davis, J. D., \& LEVINe, M. W. (1977). Model for control of ingestion. Psychological Review, 84, 379-412.

Davis, J. D., \& Perez, M. C. (1993). Food deprivation-induced and palatability-induced microstructural changes in ingestive behavior. American Journal of Physiology, 264, R97-R103.

DaVis, J. D., \& SмiтH, G. P. (1992). Analysis of the microstructure of the rhythmic tongue movements of rats ingesting maltose and sucrose solutions. Behavioral Neuroscience, 106, 217-228.

DWyer, D. M., \& QUIRK, R. H. (in press). Context conditional flavor preferences in the rat based on fructose and maltodextrin reinforcers. Journal of Experimental Psychology: Animal Behavior Processes.

Elizalde, G., \& Sclafani, A. (1988). Starch-based conditioned flavor preferences in rats: Influence of taste, calories and CS-US delay. Appetite, 11, 179-200.

GriLl, H. J., \& Norgren, R. (1978). The taste reactivity test: I. Mimetic responses to gustatory stimuli in neurologically normal rats. Brain Research, 143, 263-279.

HARRIS, J. A., \& THEIN, T. (2005). Interactions between conditioned and unconditioned flavor preferences. Journal of Experimental Psychology: Animal Behavior Processes, 31, 407-417.

HigGs, S., \& CoOper, S. J. (1998). Effects of benzodiazepine receptor ligands on the ingestion of sucrose, intralipid, and maltodextrin: An investigation using a microstructural analysis of licking behavior in a brief contact test. Behavioral Neuroscience, 112, 447-457.

Holland, P. C. (1990). Event representation in Pavlovian conditioning: Image and action. Cognition, 37, 105-131.

HsiaO, S., \& FAN, R. J. (1993). Additivity of taste-specific effects of sucrose and quinine-Microstructural analysis of ingestive behavior in rats. Behavioral Neuroscience, 107, 317-326.

Myers, K. P., \& Sclafani, A. (2001a). Conditioned enhancement of flavor evaluation reinforced by intragastric glucose: I. Intake acceptance and preference analysis. Physiology \& Behavior, 74, 481-493.

Myers, K. P., \& Sclafani, A. (2001b). Conditioned enhancement of flavor evaluation reinforced by intragastric glucose: II. Taste reactivity analysis. Physiology \& Behavior, 74, 495-505.

Nissenbaum, J. W., \& Sclafani, A. (1987). Sham-feeding response of rats to polycose and sucrose. Neuroscience \& Biobehavioral Reviews, 11, 215-222.

PeArCE, J. M. (2002). Evaluation and development of a connectionist theory of configural learning. Animal Learning \& Behavior, 30, 73-95.

Rozin, P., \& Zellner, D. (1985). The role of Pavlovian conditioning in the acquisition of food likes and dislikes. Annals of the New York Academy of Sciences, 443, 189-202. 
SCLAFAnI, A. (1987). Carbohydrate taste, appetite, and obesity-An overview. Neuroscience \& Biobehavioral Reviews, 11, 131-153.

SCLAFANI, A. (2002). Flavor preferences conditioned by sucrose depend upon training and testing methods: Two-bottle tests revisited. Physiology \& Behavior, 76, 633-644.

Sclafani, A. (2004). The sixth taste? Appetite, 43, 1-3.

Sclafani, A., \& ACKroff, K. (1994). Glucose-conditioned and fructose-conditioned flavor preferences in rats-Taste versus postingestive conditioning. Physiology \& Behavior, 56, 399-405.

Sclafani, A., Cardieri, C., Tucker, K., Blusk, D., \& Ackroff, K. (1993). Intragastric glucose but not fructose conditions robust flavor preferences in rats. American Journal of Physiology, 265, R320-R325.

Sclafani, A., Fanizza, L. J., \& Azzara, A. V. (1999). Conditioned flavor avoidance, preference, and indifference produced by intragastric infusions of galactose, glucose, and fructose in rats. Physiology \& Behavior, 67, 227-234.

Sclafani, A., \& Nissenbaum, J. W. (1988). Robust conditioned flavor preference produced by intragastric starch infusions in rats. American Journal of Physiology, 255, R672-R675.

Spector, A. C., Klumpr, P. A., \& Kaplan, J. M. (1998). Analytical issues in the evaluation of food deprivation and sucrose concentration effects on the microstructure of licking behavior in the rat. Behavioral Neuroscience, 112, 678-694.

Spector, A. C., \& ST. John, S. J. (1998). Role of taste in the microstruc- ture of quinine ingestion by rats. American Journal of Physiology, 274(6, Pt. 2), R1687-R1703.

Stevenson, R. J., BoAKes, R. A., \& WiLson, J. P. (2000). Resistance to extinction of conditioned odor perceptions: Evaluative conditioning is not unique. Journal of Experimental Psychology: Learning, Memory, \& Cognition, 26, 423-440.

Warwick, Z. S., Synowski, S. J., Coons, V., \& Hendrickson, A. (1999). Flavor-cued modulation of intake in rats: Role of familiarity and impact on 24-h intake. Physiology \& Behavior, 67, 527-532.

Warwick, Z. S., \& Weingarten, H. P. (1996). Flavor-postingestive consequence associations incorporate the behaviorally opposing effects of positive reinforcement and anticipated satiety: Implications for interpreting two-bottle tests. Physiology \& Behavior, 60, 711-715.

\section{NOTE}

1. There is no agreed term for these sustained runs of licking: For example, they were called clusters by Davis and Smith (1992) and bursts by Spector et al. (1998). I have adopted the term cluster in the present article because I have also adopted the behavioral criterion used by Davis and coworkers to define a pause in an otherwise sustained stream of licking.

(Manuscript received January 14, 2008; revision accepted for publication January 17,2008 .) 